\title{
An analysis of the impact of policies and political affiliation on racial disparities in COVID-19 infections and deaths in the USA
}

\author{
Michael A. Hamilton ${ }^{1}$ (D) Danielle Hamilton ${ }^{2} \cdot$ Oluwatamilore Soneye $^{1} \cdot$ Olorunshola Ayeyemi $^{1} \cdot$ Raed Jaradat $^{3}$
}

Received: 13 April 2021 / Accepted: 25 August 2021 / Published online: 24 September 2021

(c) The Author(s), under exclusive licence to Springer Nature Switzerland AG 2021

\begin{abstract}
This research aimed to quantify the racial disparities of COVID-19 for primarily positive tests and deaths across the US and territories individually and collectively. The first research hypothesis investigated whether positive cases and death rates were higher for people of color (POC) than the White ethnic group. The second hypothesis examined whether there is a significant difference in confirmed positive cases and death rates between ethnic groups across the US and territories. The third hypothesis investigated if political party control and governmental policies affected the number of cases and death proportion rates across ethnic groups. The research findings suggest that POC positive cases and death rates were higher in some states. Black ethnic groups were dying at a high rate in the southeastern states, the District of Columbia, and in Maryland. Specifically, in the District of Columbia, the death rate is five times higher than the White ethnic group. For Latinx ethnic groups, the high cases and death rates have mostly occurred in western states, including Texas. The Latinx ethnic group accounted for half the total deaths in Texas and California. The Latinx ethnic group death rate is higher than the White ethnic group in four states: Texas, California, New Mexico, and the District of Columbia. The research findings also show that the rate of deaths and cases per ethnic group for policies and political factors were significant except for the mask mandate policy. Based on the analyzed data, mask mandates were not a factor in the cases or death rates of any ethnic group. Each state's policies for bars, curfews, public schools, and travel—along with legislative party control—had the most influences across ethnic groups. The research results for the death rates and number of cases due to these implemented policies varied between ethnic groups.
\end{abstract}

Keywords COVID-19 $\cdot$ Infection rates $\cdot$ Death rates $\cdot$ Ethnical disparities $\cdot$ Policy $\cdot$ Political

\section{Introduction and background}

Michael A. Hamilton

mahamilton1@ncat.edu

Danielle Hamilton

daniellehamilton1000@yahoo.com

Oluwatamilore Soneye

oosoneye@aggies.ncat.edu

Olorunshola Ayeyemi

oeayeyemi@aggies.ncat.edu

Raed Jaradat

jaradat@ise.msstate.edu

1 Department of Industrial and Systems Engineering, North Carolina Agricultural and Technical State University, Greensboro, NC, USA

2 Hamilton Digital Studios LLC, Greensboro, NC, USA

3 Department of Industrial and Systems Engineering, Mississippi State University, Starkville, MS, USA
The Coronavirus Disease 2019 (COVID-19) pandemic has been devastating throughout the USA and around the world. The first cases of the virus were identified in Wuhan, China, and over 1.5 million people worldwide have died from this disease [8]. The growing rate of infection of COVID-19 in the USA, as well as the high death rates, compelled leaders to develop non-pharmaceutical interventions (NPIs) aimed at curtailing the spread of the infection. These NPIs included isolation and quarantine, social distancing, facial masks, and hand hygiene. In a country like the USA where human rights are highly regarded, the enforcement of restrictive health policies to contain the spread of the virus has been a challenging task. This can be attributed to diverse political affiliations, socioeconomic status, and demographic characteristics. As some countries such as China, Vietnam, South Korea, and Taiwan have been able to maintain low infection rates below 
$10 \%$, the opposite has been the case in the USA, Italy, and Spain [7, 20, 30].

Apart from the introduction of NPIs, some other policies were enforced to contain the spread of the virus. These policies included the closures of restaurants and bars, virtual learning in schools, lockdowns, curfews, and travel advisories. At the direction of the federal government, these policies have been enforced as the discretion of state government. The previous literature has studied the effectiveness of some of these policies on infection rate and death cases. This includes a study which examined the impact of social distancing policies on COVID-19 across different countries [7]. It was observed that these interventions were essential in slowing the spread of the virus. Communities with high population density had difficulties enforcing the NPIs, while those with low population density were able to effectively impose strict NPIs. Other studies showed that policies such as school closures have led to a reduction in the infection rate in some countries [25].

The difference in the mortality rates in countries like France and Belgium (16\%) and Singapore and Qatar $(0.1 \%)$ showed how effective timely government decisions can curb the spread of the virus. The variations in mortality rate were attributed to government effectiveness, transport infrastructure, and aging population among other factors [17]. The hesitation to implement these policies resulted in high infection and death rates [2], and the introduction of these policies, which was intended to slow the spread of the virus, harmed the economy $[11,13]$. Extended lockdowns led to the closure of non-essential businesses, thereby increasing the unemployment rate. These government policy measures were instated to minimize the spread of COVID-19, which had a significant impact on the economy and standard civic norms.

In the USA, African Americans and other people of color (POC) were dying at a higher proportional death rate than people of Caucasian descent. A reason for the higher proportional rates is that POCs tend to work in essential occupations such as retail grocery workers, public transit employees, healthcare workers, and custodial staff [12]. These types of jobs do not always observe social distancing measures, which increases the likelihood of infection for the workers. Another impact on the proportional death rates is the lack of health insurance among POC communities. Inadequate insurance decreases the probability that sick individuals would go to the hospital due to the fear of not being able to afford the cost of care. Since POCs, specifically, African Americans, are more likely to have underlying conditions such as diabetes, hypertension, and heart disease, the risk of death from COVID-19 is significantly higher than the national average [1].

The main contribution of this research is to quantify the racial disparities of COVID-19 for primarily positive tests and deaths across the US and territories individually and collectively. This includes an investigation of the policies that were implemented by each state and whether the state governmental leadership was Republican-led, Democrat-led, or equally split between the two major parties. The hypotheses for this effort are listed below:

- The first hypothesis investigates whether the positive cases and death rates for POCs were higher than the White ethnic group.

- The second hypothesis examined whether there is a significant difference in confirmed positive cases and death rates between ethnic groups across the US and territories.

- The third hypothesis investigates if political party control and governmental policies affected case and death proportion rates across ethnic groups.

The data used for obtaining the total cases and deaths based on ethnicity is provided from The COVID Tracking Project at The Atlantic (https://covidtracking.com) published under the Creative Commons CC BY 4.0 license. In collaboration with the Boston University Center for Antiracist Research, this research organization was established in April 2020, which focuses on providing testing and patient outcomes across 50 states, five territories, and the District of Columbia. As of December 2020, the COVID Tracking Project has gathered data for over 232 million COVID tests, 17 million positive cases, and 309,000 deaths due to COVID19. The dataset of interest for this effort was the COVID Racial Data Tracker (https://covidtracking.com/race). Ballotpedia provided the governors and state congress data. Ballotpedia is a digital encyclopedia that contains data about politics and political position in the American governance system [3]. The New York Times policy mandate dashboard provided the policy data for mask mandates, restaurants, and bars policies [19]. The school policy data was provided by EducationWeek, which is a news organization focus solely on K-12 educational news and information [24]. Finra provided the curfew policy data. Finra is a US Congress authorized organization that protects American investors, ensuring broker-dealer industries operate with integrity [10]. The National Academy provided the travel advisory data for State Health Policy (NASHP). NASHP is a nonpartisan forum for policymakers to innovate and recommend solutions for health policy issues [26].

\section{Research and methodology}

The COVID Racial Data Tracker (CRDT) dataset is divided into four categories. (1) Cases are referred to as the confirmed and probable cases of COVID-19; (2) Deaths are referred to as the confirmed and probable deaths due to COVID-19; 
Table 1 State political and policy factors

\begin{tabular}{lll}
\hline $\begin{array}{l}\text { Political/policy } \\
\text { factors }\end{array}$ & Levels & $\begin{array}{l}\text { State totals } \\
\text { (cases)|(deaths) }\end{array}$ \\
\hline Governor & RID & $(26 \mid 23)$ \\
State legislature & RIDISplit & $(30|18| 1) \mid(29|19| 1)$ \\
State senate & RID & $(32 \mid 17) \mid(31 \mid 18)$ \\
Mask mandate & YIN & $(40 \mid 9)$ \\
Restaurants & Bothloutdoorlclosed & $(37|8| 4) \mid(36|9| 4)$ \\
Curfew & YIN & $(31 \mid 18) \mid(32 \mid 18)$ \\
Bars & Openlclosed & $(36 \mid 13) \mid(37 \mid 12)$ \\
Public Schools & Virtuallhybrid & $(47 \mid 2)$ \\
Travel advisory & YIN & $(17 \mid 32)$ \\
\hline
\end{tabular}

(3) Hospitalizations are referred to the individuals who have been hospitalized due to COVID-19; (4) Tests are referred to the total tests (both positive and negative) conducted for COVID-19. For each of the categories, both date and state information are provided, and the 14 ethnic races are subdivided into 12 ethnic groups. The 12 groups are Whites, Blacks, Latinx, Asian, American Indian, and Alaska Native (AIAN), Native Hawaiian and Pacific Islander (NHPI), Multiracial, Other, Unknown, Hispanic Ethnicity, Non-Hispanic Ethnicity, and Ethnicity Unknown. In this research the focus was on the first six of the 12 ethnics groups since their ethnic backgrounds have been fully confirmed and the data for cases and deaths for each of the ethnic groups were evaluated. The other ethnic groups, hospitalizations, and tests were omitted from this research because the research focus was centered around cases and deaths instead of the overall hospitalizations and testing totals.

During the data collection period from April 12, 2020 to December 20, 2020, 49 states provided data on ethnicity cases and deaths. These cases and death datasets are mutually exclusive in regards to how individual states reported the information. For instance, New York did not report case data during the collection period; however, they did provide data for the death cases. The case and death datasets were provided in the cumulative form for all categories, which was converted to frequency form. The data, as shown in Table 1 , represents the political governments and policies for each state. Table 1 shows the political and policy variables and their factor levels. Afterward, the dataset was aggregated monthly per state, converting cases and deaths as proportions of the states' totals by ethnic group.

For each state congress and governor, the government majority control was either Republican, Democrat, or split evenly. The level is determined for state congress based on the party that has majority control. Governor is determined based on the party the elected official represented. Within the used dataset, twenty-six states have Republican governors, and 23 states have Democrat governors. For the state legisla-

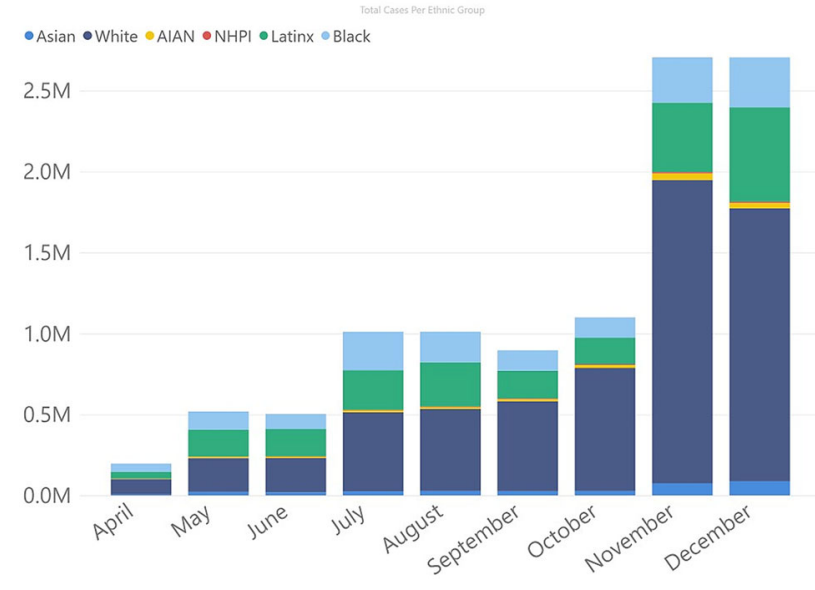

Fig. 1 Total cases per ethnic group

ture, 30 and 29 state legislatures are Republican-controlled, and 18 and 19 state legislatures are Democrat-controlled for the cases and death datasets, respectively. Alaska was the only state to have an evenly controlled state legislature. For the state Senate, 32 and 31 state Senates are Republican-controlled, while 17 and 18 state Senates are Democrat-controlled for the cases and deaths dataset. Forty states had mask mandates, and four other states closed restaurants completely. Restaurants were open entirely for 37 and 36 states, while 8 and 9 states restaurants served outdoors. Forty-seven states conducted public school education virtually, and two states used a hybrid model. State-level travel advisories were issued in 17 states, and 32 states did not have travel advisories during this time.

The dataset was transformed to provide monthly statistics of the proportion of cases and death for each state across ethnic groups. R (version 4.0.2) was used to conduct the research's data analysis with the integrated development environment RStudios. $\mathrm{R}$ is a statistical computing environment and language that Bell Laboratories developed. It provides various statistical and graphical capabilities and is an open-source product [23]. In conjunction with $\mathrm{R}$, Microsoft Power BI was used to provide the additional visualizations, along with the ggplot package [5].

\section{CRDT data analysis}

The aggregated statistics for total cases and deaths across the USA and its territories are calculated and provided followed by an evaluation of the proportionality of cases and deaths across ethnic groups and the POC rate of death compared to the White ethnic group. Figure 1 shows the total number of positive cases per ethnic group.

There were over 10.6 million cases confirmed between April 12, 2020, and December 20, 2020. Whites accounted 


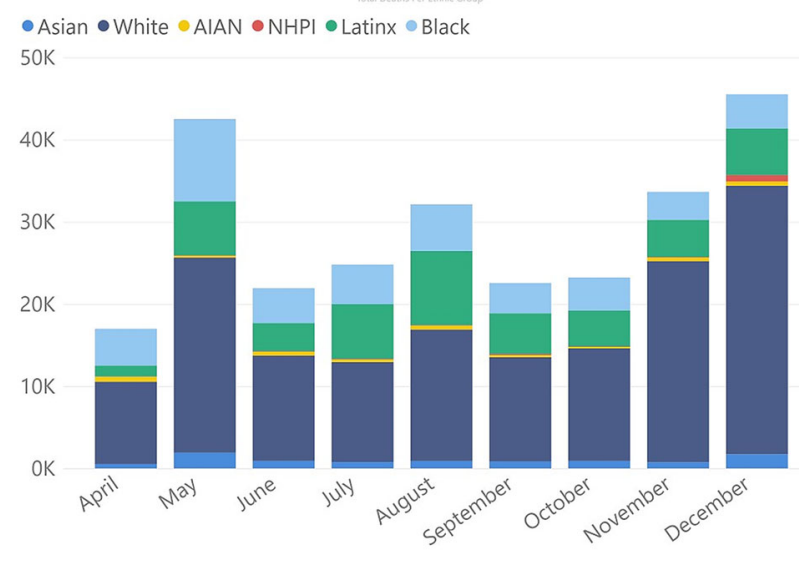

Fig. 2 Total deaths per ethnic group across the US
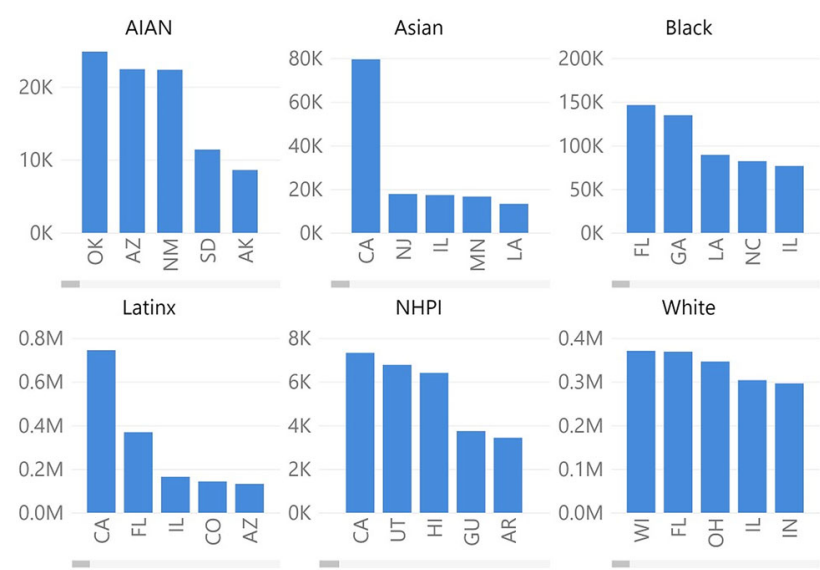

Fig. 3 Total cases per ethnic group per state/territory

for over 60 percent of the total positive cases for COVID-19. Latinxs and Blacks accounted for $21 \%$ and $14 \%$ of the total positive cases, while AIAN, Asian, and NHPI accounted for $4 \%$ together. The total number of deaths per ethnic group is shown in Fig. 2.

There were 263,298 deaths confirmed between April 12, 2020, and December 20, 2020. Whites accounted for over 60 percent of the confirmed deaths for COVID-19. Latinxs and Blacks accounted for $17 \%$ of confirmed deaths, while AIAN, Asian, and NHPI accounted for $5 \%$ together. In Fig. 3, the data is aggregated based on the total number of positive cases by each ethnic group across the states and territories individually.

California had the most confirmed cases for Asian, Latinx, and NHPI ethnic groups. Florida, Wisconsin, and Oklahoma had the most confirmed cases for Blacks, Whites, and AIAN cases, respectively. California, Illinois, Louisiana, and Florida are in the top five of multiple ethnic groups for most positive confirmed cases. Figure 4 shows the total number of confirmed deaths by each ethnic group across the states and territories.

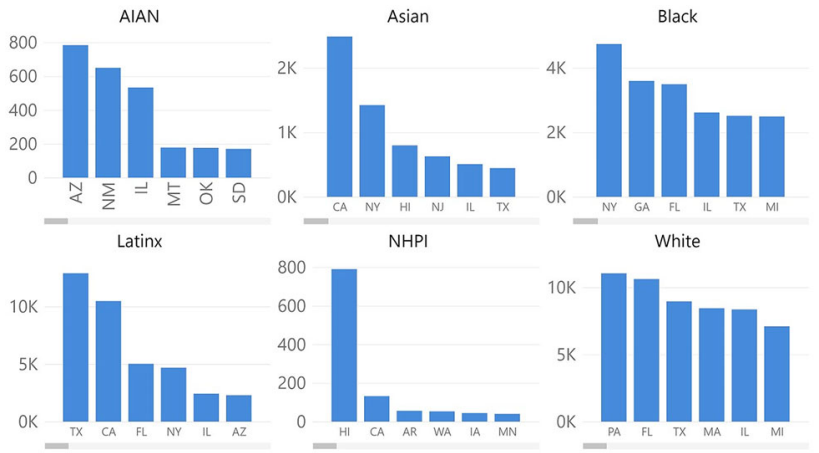

Fig. 4 Total deaths per ethnic group per state/territory

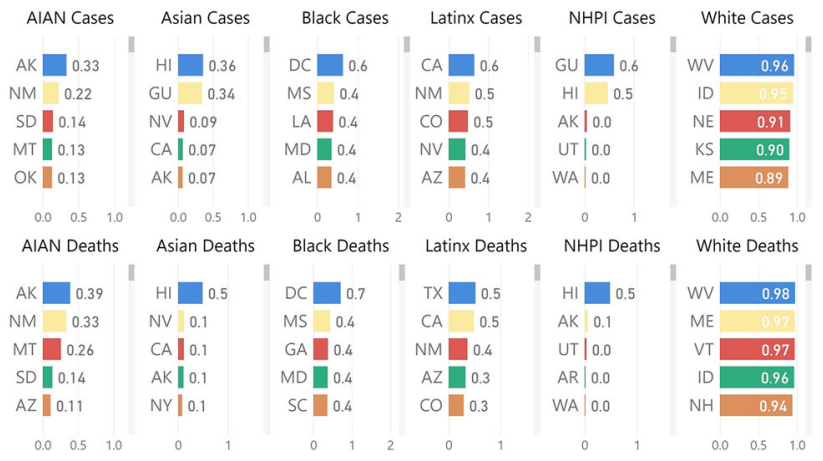

Fig. 5 Proportional rate of cases and deaths

The states with the most confirmed deaths for AIAN, Asians, Blacks, Latinxs, NHPIs, and Whites were Arizona, California, New York, Texas, Hawaii, and Pennsylvania. California, New York, Illinois, Florida, Texas, and Hawaii were in the top five for multiple ethnic groups for most confirmed deaths due to COVID-19. To better understand each state's rate of cases and deaths for ethnic groups, proportional analyses were conducted. Figure 5 shows proportional results of total cases and deaths across ethnic groups.

The states with the highest proportions of positive cases across ethnic groups were Alaska, Hawaii, District of Columbia, California, Guam, and West Virginia. The US 2020 Census data was used to provide the overall ethnic population percentage for the top five states with the largest proportions of cases and deaths for each ethnic group. The census percentage per specified ethnic group is denoted with an ' $*$ ' by the state name [29]. The results show that AIAN accounted for $33 \%, 22 \%, 14 \%, 13 \%$, and $13 \%$ of the positive cases in Alaska (*15.6\%), New Mexico (*11\%), South Dakota $(* 9 \%)$, Montana $(* 6.7 \%)$, and Oklahoma $(* 9.4 \%)$, respectively. Asian accounted for $36 \%, 34 \%, 9 \%, 7 \%$, and $7 \%$ of the positive cases in Hawaii $(* 37.6 \%)$, Guam $(* 32.5 \%)$, Nevada $(* 8.7 \%)$, California $(* 15.5 \%)$, and Alaska $(* 6.5 \%)$, respectively. Blacks accounted for $64 \%, 42 \%, 39 \%, 36 \%$, and $35 \%$ of the positive cases in the District of Columbia (*46\%), Mississippi (*37.8\%), Louisiana (*32.8\%), Mary- 
land $(* 31.1 \%)$, and Alabama $(* 26.8 \%)$, respectively. Latinxs accounted for $65 \%, 52 \%, 49 \%, 42 \%$, and $42 \%$ of the total cases in California $(* 39.4 \%)$, New Mexico $(* 49.3 \%)$, Colorado $(* 21.8 \%)$, Nevada $(* 29.2 \%)$, and Arizona $(* 31.7 \%)$, respectively. NHPI accounted for $60 \%, 47 \%, 5 \%, 3 \%$, and $2 \%$ of the cases in Guam $(* 44.6 \%)$, Hawaii $(* 10.1 \%)$, Alaska $(* 1.4 \%)$, Utah $(1.1 \%)$, and Washington $(* 0.8 \%)$, respectively. Whites accounted for $96 \%, 95 \%, 91 \%, 90 \%$, and $89 \%$ of the cases in Montana (*88.9\%), Idaho (*93\%), Nebraska $(* 88.1 \%)$, Kansas $(* 86.3 \%)$, and Maine $(* 94.4 \%)$ respectively.

The states with the highest proportions of confirmed deaths across ethnic groups were Alaska, Hawaii, District of Columbia, Texas, Hawaii, and West Virginia. AIANs accounted for $39 \%, 33 \%, 26 \%, 14 \%$, and $11 \%$ of the confirmed deaths in Alaska (*15.6\%), New Mexico (*11\%), Montana $(* 6.7 \%)$, South Dakota $(* 9 \%)$, and Oklahoma $(* 9.4 \%)$, respectively. Asians accounted for $49 \%, 12 \%, 12 \%$, $11 \%$, and $8 \%$ of the confirmed deaths in Hawaii $(* 37.6 \%)$, Nevada $(* 8.7 \%)$, California $(* 15.5 \%)$, Alaska $(* 6.5 \%)$, and New York $(* 9 \%)$ respectively. Blacks accounted for $71 \%$, $44 \%, 38 \%, 37 \%$, and $37 \%$ of the confirmed deaths in the District of Columbia (*46\%), Mississippi (*37.8\%), Georgia (*32.6\%), Maryland (*31.1\%), and South Carolina (*27\%), respectively. Latinxs accounted for 52\%, 49\%, 37\%, 33\%, and $29 \%$ of the confirmed deaths in Texas $(* 39.7 \%)$, California $(* 39.4 \%)$, New Mexico $(* 49.3 \%)$, Arizona $(* 31.7 \%)$, and Colorado $(* 21.8 \%)$, respectively. NHPIs accounted for $49 \%, 6 \%, 4 \%, 2 \%$, and $2 \%$ of confirmed deaths in Hawaii $(* 10.1 \%)$, Alaska $(* 1.4 \%), \operatorname{Utah}(* 1.1 \%)$, Arkansas $(* 0.4 \%)$, and Washington $(* 0.8 \%)$, respectively. Whites accounted for $98 \%, 97 \%, 97 \%, 96 \%$, and $94 \%$ of the deaths in West Virginia $(* 92 \%)$, Maine $(* 93 \%)$, Vermont $(* 92.6 \%)$, Idaho $(* 81.6 \%)$, and New Hampshire (*89.8\%).

The final proportional analysis conducted is called the People of Color Death Rate (POCDR). It is defined as a proportion of death of a given POC divided by the proportion of Whites' deaths for a given state. A POCDR value of one means that the POC death rate is equal to the White death rate. Values greater than one indicate the death rate is higher than the White death rate, and values lower than one indicate the POC death rate is less than the White death rates. The ranked order results for the top five POCDR scores for the POC ethnic groups are shown in Fig. 6.

The results show that the AIAN death rate is $1.19 \%$ higher than the White deaths in New Mexico. In other words, for every ten Whites' deaths, there would be approximately 11.9 $(10 * 1.19)$ AIAN deaths. Asian and NHPIs were dying at a 30 to 1 ratio in Hawaii. Blacks were dying at approximately a 6 to 1 ratio in the District of Columbia. Latinxs were dying at around a 1.5 to 1 ratio in California, Texas, New Mexico, and the District of Columbia. A clustering analysis using kmeans clustering was performed to observe states' clusters

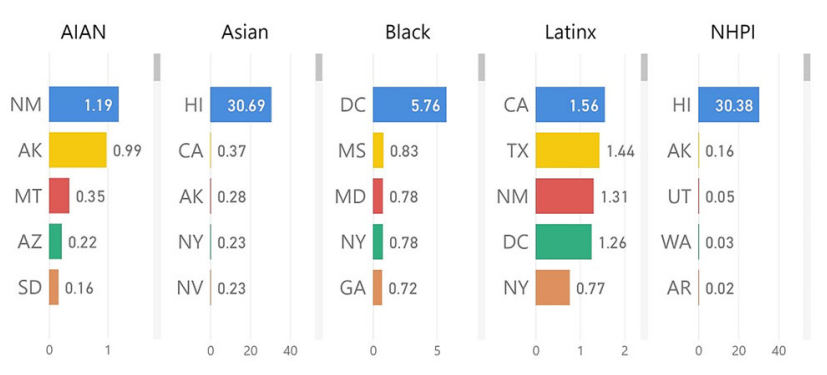

Fig. 6 POCDR scores

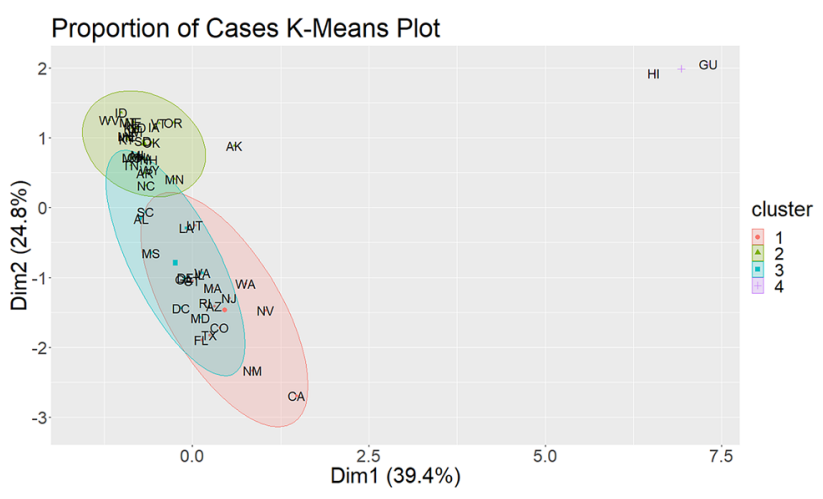

Fig. 7 Prop. of case K-means plot

with similar proportions of positive cases and deaths across the ethnic groups. K-means clustering is an unsupervised machine learning algorithm that groups datasets together that have high intra-class similarities. Each cluster is represented by a centroid, which is the clustered dataset [18]. For the proportion of confirmed cases and deaths analyses, the "factoextra" package from the Comprehensive R Archive Network (CRAN) repository was performed to determine the number of clusters required for the evaluation [15]. The "gap statistic" approach was used to determine the number of clusters by comparing change within-cluster dispersion with the appropriate reference null distribution [27]. The results determined that the optimal number of clusters was four clusters for the proportion of cases and three clusters for the proportion of deaths. The k-means was configured to obtain 25 different random starting assignments and select the best results based on the one with the lowest within-cluster variation. Each observation was assigned to their closest centroid using the Euclidean distance approach.

The proportion of confirmed cases across ethnic groups per state is shown in Fig. 7 and Table 2.

Cluster 1 states are AZ, CA, CO, CT, FL, IL, MA, NJ, NM, NV, RI, TX, UT, and WA. Cluster 2 states are AK, AL, IA, ID, IN, KS, KY, ME, MI, MN, MO, MT, NC, ND, NE, NH, OH, OK, OR, PA, SD, TN, VT, WI, WV, and WY. Cluster 3 states are AL, DC, DE, GA, LA, MD, MS, SC, and VA. Cluster 4 states/territories are $\mathrm{HI}$ and GU. 
Table 2 Prop. of cases K-means centers

\begin{tabular}{llllllll}
\hline Cluster & AIAN & Asian & Black & Latinx & NHPI & White & $\begin{array}{l}\text { Cluster } \\
\text { size }\end{array}$ \\
\hline 1 & 0.03 & 0.03 & 0.09 & 0.40 & 0.00 & 0.44 & 14 \\
2 & 0.04 & 0.02 & 0.09 & 0.01 & 0.00 & 0.83 & 26 \\
3 & 0.00 & 0.02 & 0.37 & 0.09 & 0.00 & 0.51 & 9 \\
4 & 0.00 & 0.35 & 0.01 & 0.00 & 0.53 & 0.10 & 2 \\
\hline
\end{tabular}

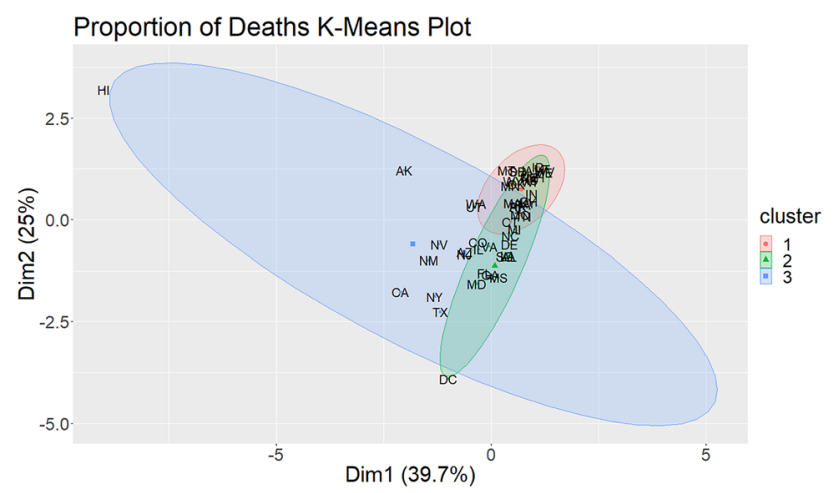

Fig. 8 Prop. of deaths K-means plot

Table 3 Prop. of cases K-means centers

\begin{tabular}{llllllll}
\hline Cluster & AIAN & Asian & Black & Latinx & NHPI & White & $\begin{array}{l}\text { cluster } \\
\text { size }\end{array}$ \\
\hline 1 & 0.03 & 0.02 & 0.07 & 0.03 & 0.00 & 0.85 & 27 \\
2 & 0.01 & 0.02 & 0.35 & 0.06 & 0.00 & 0.57 & 12 \\
3 & 0.08 & 0.10 & 0.09 & 0.27 & 0.05 & 0.41 & 11 \\
\hline
\end{tabular}

The evaluation of clusters can be conducted by investigating the rate of case differences between groups. The key result from the analysis is that for the AIAN ethnic group, the mean rate of positive cases is $1.3(0.04 / 0.03)$ times higher in cluster 2 states when compared to cluster 1 states. For Asians, the cluster 4 states' mean percentage of positive cases is 11 to 17 times higher than the other clusters states. For Blacks, cluster 3 states' mean rate of positive cases is four times higher than cluster 1 and 2 states. For Latinxs, cluster 1 states' mean rate of positive cases is four times higher than cluster 3 states and 40 times higher than cluster 2 states. For Whites, cluster 2 states' mean rate of positive cases is 1.8 times higher than cluster 1 states. NHPI positive test rates were only found in Hawaii and Guam. The proportion of confirmed deaths across ethnic groups per state is shown in Fig. 8 and Table 3.

Cluster 1 states are AR, CT, IA, ID, IN, KS, KY, MA, ME, MN, MO, MT, NE, NH, OH, OK, OR, PA, RI, SD, TN, UT, VT, WA, WI, WV, and WY. Cluster 2 states are AL, DC, DE, GA, IL, LA, MD, MI, MS, NC, SC, and VA. Cluster 3 states are AK, AZ, CA, CO, FL, HI, NJ, NM, NV, NY, and TX. For

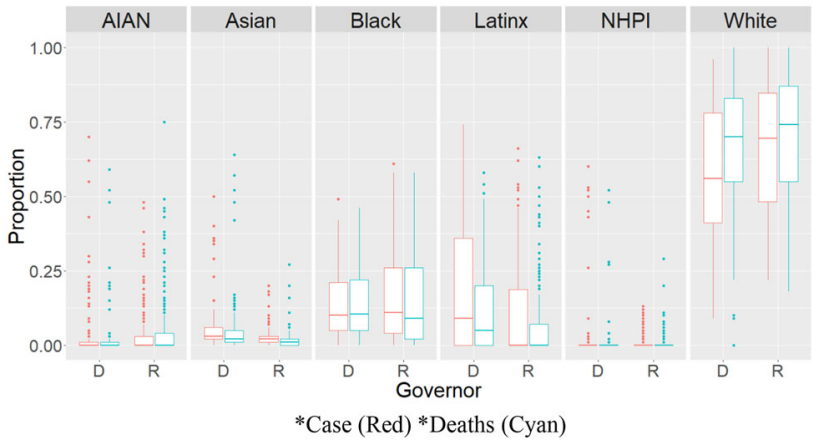

Fig. 9 Case and death proportions by governor affiliation

the AIAN ethnic group, the cluster 3 states' mean death rate is 2.6 times higher than cluster 1 states. For Asians, cluster 3 states' mean death rate is five times higher than the other clusters states. For Blacks, cluster 2 states' death rate is 3.8 and 5 times higher than cluster 3 and 1 states, respectively. For Latinxs, cluster 3 states' mean death rate is 4.5 and 9 times higher than cluster 2 and 1 states, respectively. The mean death rate for the NHPI ethnic group could not be compared between other cluster states since no deaths were reported in those clusters. For the Whites, cluster 1 states' death rate is 1.5 and 2 times higher than cluster 2 and cluster 3 states.

\section{Political and policy factors data analysis}

The impact of political and policy factors on the country's case and death rates are discussed next by evaluating the dispersion and skewness of the death and case rates across the factors, and conducting a variance analysis between the factor levels. The death and case proportions rates are reported monthly for each state.

\section{A. Data Dispersion and Skewness}

The boxplot depicted in Fig. 9 shows the monthly state cases and death proportions by governor's political affiliation.

The AIAN, Asian, and NHPI box plots are relatively short and close to zero, suggesting that most states had very few reported cases. The average median of case proportions of the three ethnic groups was $2.8 \%$. The results show minor differences between governor affiliations between these three groups. For the Black ethnic group, the Republican governorled states' case proportions dispersion is larger than the Democrat governor-led states. The results do not suggest a difference between groups; however, the difference in case proportions means and medians suggests that the case proportions are top skewed. For the Latinx, the Democrat governor-led states' case proportion dispersion is larger than 


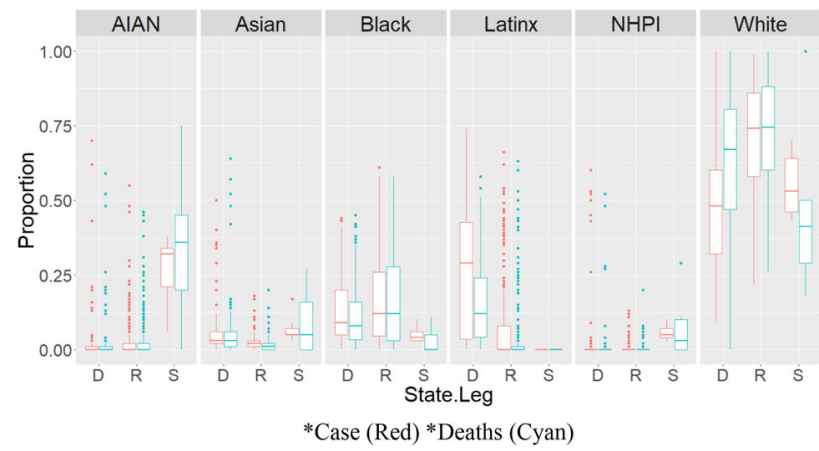

Fig. 10 Case and death proportions by state legislature majority control

the Republican-led states. The results show that the data is top skewed for both governor affiliation groups. The White ethnic group is different from the other ethnic groups; however, the dispersion between governor affiliations seems similar. The death proportions data dispersions are similar to the case proportion results. The AIAN, Asians, and NHPI box plots results are short due to the limited amount of data across the states and territories. The Black and Latinx data are top skewed, which means the data dispersion for Republican governor-led states is larger for Blacks, and Democrat-led states are larger for Latinx. White ethnic groups' death proportions suggest a mean group difference from the other ethnic groups. Figure 10 shows the case and death proportions by state legislature affiliation majority control.

Alaska is the only state with a split legislature, so the AIAN split legislature group is different from the Democrat and Republican majority-controlled groups. A large part of the data provided for this ethnic group is from Alaska. The Asian and NHPI boxplots are relatively short across all groups that suggest a high level of similarity in the case proportions between states. The case proportions are greater in Republican majority-controlled legislatures for Black ethnic groups and larger in Democrat-controlled legislatures for Latinx ethnic groups. For both groups, the case proportion rates are top skewed for the Democrat and Republican majorities. Case proportions results for White ethnic groups are likely substantially different from the other ethnic groups. Within the group, the Republican majority state legislatures' group case proportions are larger than the other two factor levels. A large proportion of the deaths of the AIAN ethnic group is from Alaska. Alaska's AIAN death proportions group seems to be significantly larger than the Republican- and Democrat-controlled state legislatures. The death proportions data dispersion for the Asian ethnic group in Alaska is larger than the other state legislature majorities. Republican-controlled legislatures have a higher mean death rate than Democrat-controlled states for the Black ethnic group. For the Latinx ethnic groups, Democratcontrolled legislatures mean death proportion rates are higher

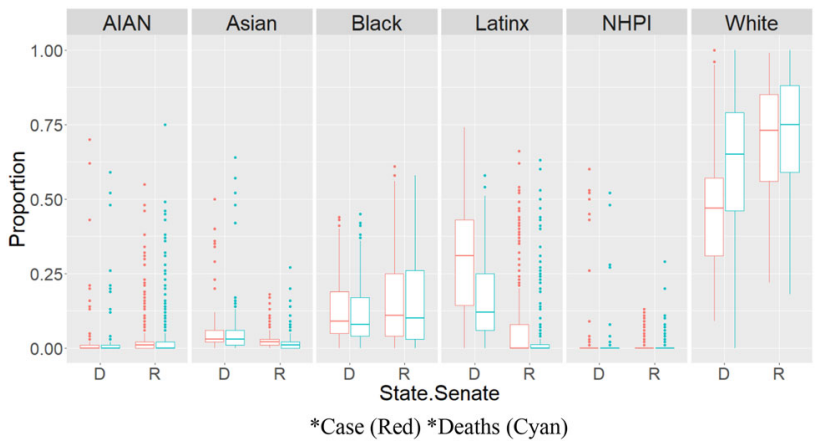

Fig. 11 Case and death proportions by state senate majority control

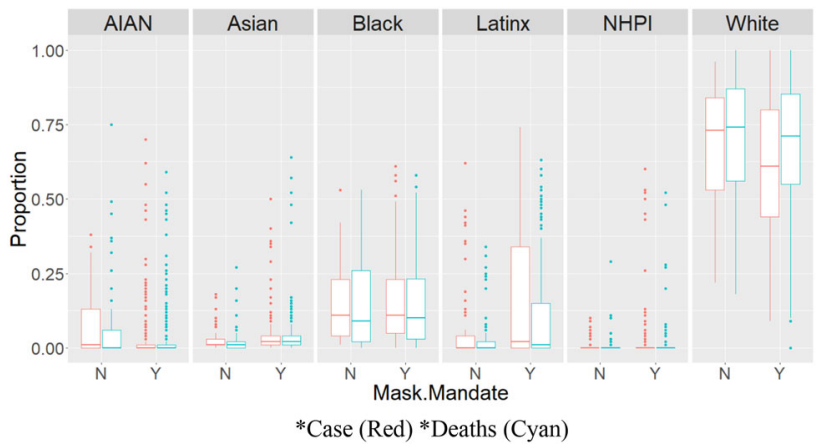

Fig. 12 Case and death proportions by mask mandate policy

than Republican-controlled legislatures. For the White ethnic group, Alaska's death proportion group seems to be significantly different from the other state legislature majorities. Figure 11 shows the case and death proportion by Senate affiliation majority control.

For the AIAN, Asian, and NHPI groups, the case proportions are relatively small and similar across Senate majority control. The Republican-controlled Senate case proportions data dispersion is larger than the Democrat-controlled Senate for the Black ethnic group. For the Latinx and White ethnic groups, the Democrat Senate majority case proportion rates seem to be substantially different from the Republicancontrolled Senate majority. For the AIAN, Asian, and NHPI groups, the death proportions are relatively small and similar across Senate majority control. For the Black ethnic group, the Republican-controlled Senate death proportions data dispersion is larger than the Democrat-controlled Senate. The Democrat-controlled Senate death proportion groups seem to be substantially different from the Republican-controlled Senate for the Latinx ethnic group. Overall, the White ethnic group death proportions are different from the other ethnic groups. Within this group, the Republican-controlled Senate data dispersion is larger than the Democrat Senate-controlled states. Figure 12 shows the case and death proportion by mask mandate policy.

For the Asian, NHPI, and Black ethnic groups, there was relatively no difference in the dispersion of case proportions 


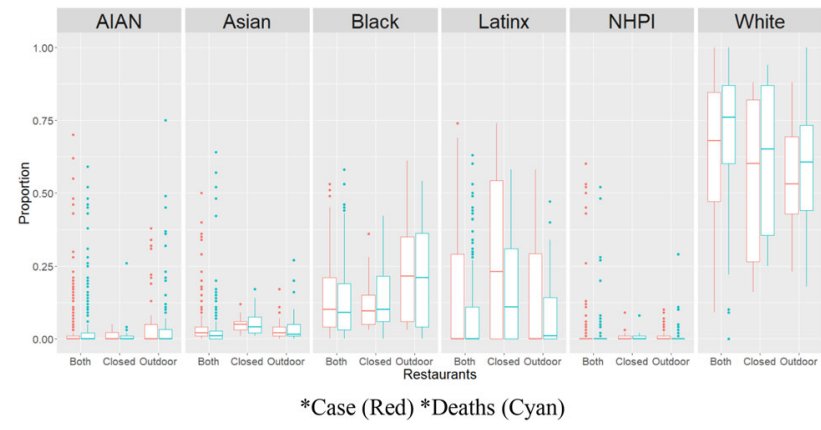

Fig. 13 Case and death proportions by restaurant policy

between mask mandate policies. AIAN and White ethnic groups' case proportions data dispersion were higher for states that did not have a mask mandate policy. For the Latinx ethnic group, the case proportion data spread was larger for states with a mask mandate policy. The data dispersion of death proportions within each ethnic group was similar to the case proportion results. The data dispersion of death proportions was larger for states with no mask policy for the Black and White ethnic groups. For the Latinx group, the data spread was larger for states with a mask mandate policy. Figure 13 shows the case and death proportion by restaurant policy.

The data dispersion of case proportion for AIAN, Asian, and NHPI was similar regardless of the restaurant policy. The Black ethnic group case proportions data spread was higher for states that implemented outdoor-only restaurant policies than the other policy groups. The lowest case proportions data spread when the restaurants were closed. For the Latinx and White ethnic groups, the largest data dispersion of case proportions was for states that closed the restaurants. However, the Latinx ethnic group yielded similar case proportions for the other two restaurant policies. No policy resulted in a larger data dispersion of case proportions than having an outdoor restaurant policy for the White ethnic group. Similar to the case proportion results, AIAN, Asian, and NHPI data dispersion of death proportion are not substantially different based on the restaurant policy. The data dispersion of death proportions was larger for restaurants with outdooronly policies for the Black ethnic group. For the Latinx and White ethnic groups, death proportions' data dispersion was larger when the restaurants were closed. However, the data dispersion rate was higher when there was no restaurant policy for the white ethnic group. Figure 14 shows the case and death proportions result from the curfew policy.

For all ethnic groups, the data dispersion of case proportions was not substantially different based on the curfew policy. However, the data spread is top skewed for the AIAN, Black, and Latinx ethnic groups. For AIAN, Asian, NHPI, Latinx, and White ethnic groups, the data dispersion for death proportions are not substantially different based on the cur-

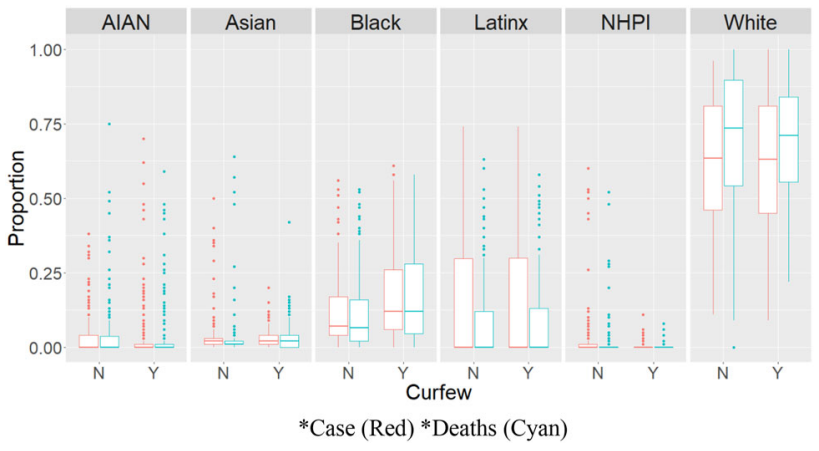

Fig. 14 Case and death proportions by curfew policy

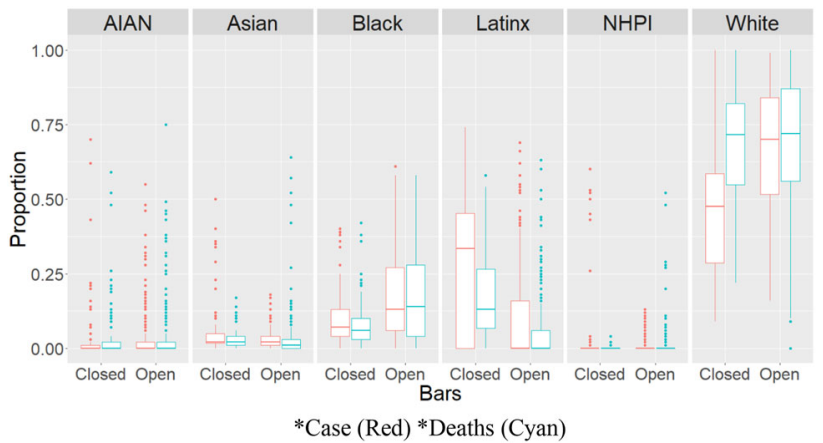

Fig. 15 Case and death proportions by bar policy

few policy. For the Black ethnic group, the data dispersion for death proportions is higher with states with a curfew policy. Figure 15 shows the case and death proportions by bar policy.

For AIAN, Asian, and NHPI ethnic groups, the data dispersion of case proportions is not substantially different based on the state's bar policy. The data dispersion rates were higher for the Black and White ethnic groups for states with open bars. For the Latinx ethnic group, the data dispersion rates were higher for states with closed bars. The data dispersion of case proportion of the White ethnic group has a substantial group difference between the state's bar policy. The data dispersion of death proportions is not substantially different based on bar policy for the AIAN, Asian, NPHI, and White ethnic groups. The dispersion rates are larger for Black ethnic groups for states with open bar policies. The dispersion rates are substantially larger for Latinx ethnic groups for states with closed bar policies. Figure 16 shows the case and death proportion results based on public school policy.

For the AIAN and Black ethnic groups, the results show a substantial difference in data dispersion case proportions groups regarding public school policy. For Asian and NHPI ethnic groups, the data dispersion rates were similar regardless of the public school policy. The data dispersion rate for the Latinx ethnic is larger for the virtual school policy. Even though the White ethnic groups are not substantially different based on policy, states that use the hybrid policy 


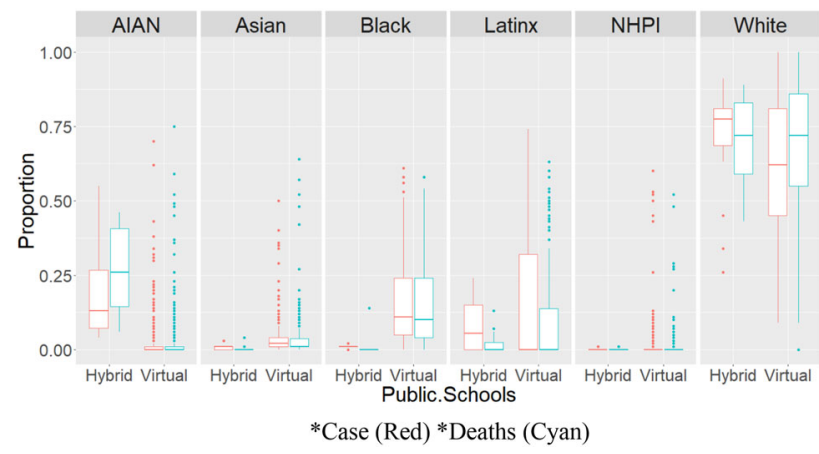

Fig. 16 Case and death proportions by public school policy

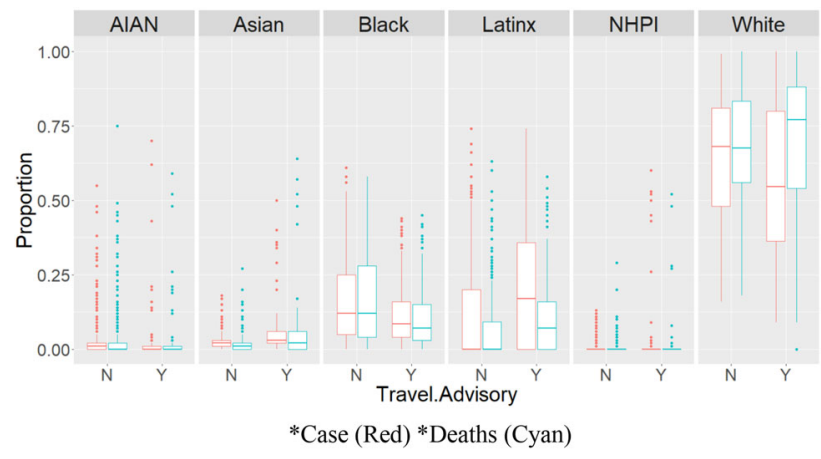

Fig. 17 Case and death proportions by travel advisory policy

dispersion variance are much smaller than the virtual policy states. The AIAN and Black ethnic groups' data dispersion of death proportions are substantially different between public school policies. The Asian, NHPI, and White ethnic groups data dispersion of death proportions are not substantially different between public school policies. The data dispersion of death proportions is larger for the virtual policy for the Latinx ethnic group. Figure 17 shows the case and death proportions by travel advisory policy.

The data dispersion of case proportions for the AIAN, Asian, and NHPI ethnic groups is not substantially different from the travel advisory. The data dispersion of case proportions was higher for states with no travel policies for the Black and White ethnic groups. For the Latinx group, the dispersion of case proportions was higher for states with travel restriction policies. The data dispersion of death proportions for the AIAN, Asian, and NHPI ethnic groups is not substantially different from the travel advisory. The dispersion of death proportions was higher for states with no travel policies for the Black ethnic group. For the Latinx and White ethnic groups, the data dispersion of case proportions was higher for states with travel restriction policies.

\section{B. Analysis of Variance}

The analysis of variance is performed to investigate the impact of political and policy factors on the percentage of cases and deaths across ethnic groups. Since the dataset was not normally distributed, the beta distribution was used since it expects the dependent variable to vary between 0 and 1 . The "betareg" package from the CRAN repository was used to conduct the analysis, which evaluated the primary factors and two-way interactions [9]. For this analysis, the main factors' results are reported, and the two-way interaction was omitted. The Tukey test was used to compare the group means of the political and policy main factors on the ethnic groups' case and death proportions. Table 4 shows the beta analysis of variance results for the case proportions across ethnic groups.

There was a significant difference in the bar policy group means for the AIAN, Black, Latinx, and White ethnic groups. For the AIAN ethnic group, the Tukey results showed that case proportions for states with closed bar policies $(M=$ $0.25, \mathrm{SD}=0.04)$ group means were significantly larger than the states with an open bar policy $(M=0.21, \mathrm{SD}=0.04)$. For the Black ethnic group, the Tukey results showed that case proportions for states with open bars $(M=0.09, \mathrm{SD}=$ $0.01)$ group mean was significantly larger than states with closed bars $(M=0.05, \mathrm{SD}=0.01)$. For the Latinx ethnic group, the Tukey results showed that case proportions for states with closed bars $(M=0.17, \mathrm{SD}=0.05)$ group means were significantly larger than states with open bars $(M=$ $0.13, \mathrm{SD}=0.04)$. For the White ethnic group, the Tukey results showed that case proportions for states with open bars $(M=0.58, \mathrm{SD}=0.07)$ group means were significantly larger than states with closed bars $(M=0.52, \mathrm{SD}=0.07)$.

There was a significant difference in the curfew policy group for Asian, Blacks, NHPI, and White ethnic groups. For the Asian ethnic group, the Tukey results showed that case proportions for states without a curfew policy $(M=0.05$, SD $=0.01)$ group means were significantly higher than states with a curfew policy $(M=0.03, \mathrm{SD}=0.01)$. For the Black ethnic group, the Tukey results showed that case proportions for states with a curfew policy $(M=0.08, \mathrm{SD}=0.01)$ group means were significantly higher than states without a curfew policy $(M=0.06, \mathrm{SD}=0.01)$. For the NHPI ethnic group, the Tukey results showed that case proportions for states without a curfew policy $(M=0.04, \mathrm{SD}=0.02)$ group means were significantly higher than states with a curfew policy $(M=$ $0.03, \mathrm{SD}=0.01)$. For the White ethnic group, the Tukey results showed that case proportions for states with a curfew policy $(M=0.58, \mathrm{SD}=0.07)$ group means were significantly higher than states without a curfew policy $(M=0.53, \mathrm{SD}=$ 0.07).

There was a significant difference in the governor's political affiliation group for the AIAN and Asian ethnic groups. For the AIAN ethnic group, the Tukey results showed that case proportions for states with a Democrat governor $(M=$ $0.25, \mathrm{SD}=0.04)$ group means were significantly higher than states with a Republican governor $(M=0.21, \mathrm{SD}=0.04)$. For the Asian ethnic group, the Tukey results showed that 
Table 4 Case proportions beta analysis of variance results

\begin{tabular}{|c|c|c|c|c|c|c|c|c|c|}
\hline \multicolumn{4}{|l|}{ AIAN } & \multicolumn{3}{|c|}{ Asian } & \multicolumn{3}{|c|}{ Black } \\
\hline Source & $d f$ & $F$ ratio & $p$-value & $d f$ & $F$ ratio & $p$-value & $d f$ & $F$ ratio & $p$-value \\
\hline Bars & 1 & 6.03 & $p<0.05$ & 1 & 1.36 & 0.244 & 1 & 36.20 & $p<0.001$ \\
\hline Curfew & 1 & 1.80 & 0.18 & 1 & 13.77 & $p<0.001$ & 1 & 12.62 & $p<0.001$ \\
\hline Governor & 1 & 5.28 & $p<0.05$ & 1 & 7.43 & $p<0.01$ & 1 & 0.06 & 0.809 \\
\hline $\begin{array}{l}\text { Mask } \\
\text { mandate }\end{array}$ & 1 & 3.81 & 0.051 & 1 & 2.70 & 0.100 & 1 & 2.69 & 0.101 \\
\hline $\begin{array}{l}\text { Public } \\
\text { schools }\end{array}$ & 1 & 70.03 & $p<0.001$ & 1 & 9.99 & $p<0.01$ & 1 & 119.30 & $p<0.001$ \\
\hline Restaurants & 2 & 0.47 & 0.626 & 2 & 2.23 & 0.108 & 2 & 20.35 & $p<0.001$ \\
\hline State leg & 2 & 33.68 & $p<0.001$ & 2 & 10.26 & $p<0.001$ & 2 & 13.05 & $p<0.001$ \\
\hline State senate & 1 & 2.81 & 0.093 & 1 & 8.58 & $p<0.01$ & 1 & 1.45 & .229 \\
\hline $\begin{array}{l}\text { Travel } \\
\text { advisory }\end{array}$ & 1 & .67 & 0.413 & 1 & 10.67 & $p<0.01$ & 1 & 16.28 & $p<0.001$ \\
\hline \multicolumn{4}{|l|}{ Latinx } & \multicolumn{3}{|c|}{ NHPI } & \multicolumn{3}{|c|}{ White } \\
\hline Source & $d f$ & $F$ ratio & $p$-value & $d f$ & $F$ ratio & $p$-value & $d f$ & $F$ ratio & $p$-value \\
\hline Bars & 1 & 4.16 & $p<0.05$ & 1 & 0.10 & 0.755 & 1 & 4.94 & $p<0.05$ \\
\hline Curfew & 1 & 0.04 & 0.836 & 1 & 7.53 & $p<0.01$ & 1 & 5.58 & $p<0.05$ \\
\hline Governor & 1 & 1.64 & 0.201 & 1 & 0.43 & 0.511 & 1 & 1.94 & 0.163 \\
\hline Mask mandate & 1 & 0.26 & 0.611 & 1 & 1.18 & 0.278 & 1 & 0.31 & 0.577 \\
\hline Public schools & 1 & 1.19 & 0.276 & 1 & 0.43 & 0.514 & 1 & 0.34 & 0.562 \\
\hline Restaurants & 2 & 2.80 & 0.061 & 2 & 0.49 & 0.614 & 2 & 13.11 & $p<0.001$ \\
\hline State leg & 2 & 3.39 & $p<0.05$ & 2 & 5.12 & $p<0.01$ & 2 & 0.80 & 0.448 \\
\hline State senate & 1 & 4.94 & $p<0.05$ & 1 & 0.01 & 0.942 & 1 & 4.18 & $p<0.05$ \\
\hline Travel advisory & 1 & 17.50 & $p<0.001$ & 1 & 1.27 & 0.260 & 1 & 10.94 & $\mathrm{p}<0.001$ \\
\hline
\end{tabular}

case proportions for states with a Democrat governor $(M=$ $0.05, \mathrm{SD}=0.01)$ group means were significantly larger than states with a Republican governor $(M=0.04, \mathrm{SD}=0.01)$.

There was a significant difference in the public school policy group means for the AIAN, Asian, and Black ethnic groups. For the AIAN group, the Tukey results showed that the case proportions for states that use a hybrid learning $(M$ $=0.37, \mathrm{SD}=0.07)$ group means were significantly larger than states that used virtual learning $(M=0.09, \mathrm{SD}=0.02)$. For the Asian ethnic group, the Tukey results showed that the case proportions for states that use virtual learning $(M$ $=0.05, \mathrm{SD}=0.01)$ group means were significantly larger than states that used hybrid learning $(M=0.05, \mathrm{SD}=0.01)$. For the Black ethnic group, the Tukey results showed that the case proportions for states that use virtual learning $(M=$ $0.11, \mathrm{SD}=0.01)$ group means were significantly larger than states that used hybrid learning $(M=0.02, \mathrm{SD}=0.01)$.

There was a significant difference in the restaurant policy group means for the Black and White ethnic groups. For the Black ethnic group, the Tukey results showed that the case proportions for states that had outdoor-only restaurant policies $(M=0.10, \mathrm{SD}=0.01)$ group means were significantly larger than states with no policy $(M=0.04, \mathrm{SD}=0.01)$ or the restaurants were closed $(M=0.05, \mathrm{SD}=0.01)$. For the
White ethnic group, the Tukey results showed that the case proportions for states that had outdoor-only restaurant policies $(M=0.46, \mathrm{SD}=0.09)$ group means were significantly smaller than states with no policy $(M=0.59, \mathrm{SD}=0.07)$ or the restaurants were closed $(M=0.62, \mathrm{SD}=0.09)$.

There was a significant difference in state legislature party majority group means for the AIAN, Asian, Black, Latinx, and NHPI ethnic groups. For the AIAN ethnic group, the Tukey results showed that the case proportions in states with a split state legislature $(M=0.45, \mathrm{SD}=0.10)$ group means were significantly larger than Democrat $(M=0.13, \mathrm{SD}=$ $0.05)$ and Republican $(M=0.10, \mathrm{SD}=0.03)$ majoritycontrolled legislatures. For the Asian ethnic group, the Tukey results showed that the case proportions in states with a split $(M=0.06, \mathrm{SD}=0.03)$ and Democrat $(M=0.05, \mathrm{SD}=0.01)$ majority-controlled state legislature group means were significantly larger than Republican $(M=0.02, \mathrm{SD}=<0.01)$ majority-controlled legislatures. For the Black ethnic group, the Tukey results showed that the case proportions in states with a Republican $(M=0.09, \mathrm{SD}=0.02)$ or Democrat $(M=0.08, \mathrm{SD}=0.02)$ majority-controlled state legislature group means are significantly larger than split $(M=$ $0.03, \mathrm{SD}=0.02$ ) controlled state legislatures. For the Latinx ethnic group, the Tukey results showed did not reveal a 
significant difference between group means for the adjusted p-value factor level comparisons even though family p-value was found to be significant. For the NHPI ethnic group, the Tukey results showed that the case proportions in states with a split state legislature $(M=0.08, \mathrm{SD}=0.05)$ group means were significantly larger than Democrat $(M=0.02, \mathrm{SD}=$ $0.01)$ and Republican $(M=0.01, \mathrm{SD}=<0.01)$ majoritycontrolled legislatures.

There was a significant difference in state Senate legislature party majority group means for the Asian, Latinx, and White ethnic groups. For the Asian ethnic group, the Tukey results showed that the case proportions in states with a Republican-controlled Senate $(M=0.06, \mathrm{SD}=0.02)$ group means were significantly larger than Democrat-controlled Senates $(M=0.03, \mathrm{SD}=0.01)$. For the Latinx ethnic group, the Tukey results showed that the case proportions in states with a Democrat-controlled Senate $(M=0.21, \mathrm{SD}=$ $0.08)$ group mean are significantly larger than Republicancontrolled Senates $(M=0.10, \mathrm{SD}=0.04)$. For the White ethnic group, the Tukey results showed that the case proportions in states with a Republican-controlled Senate $(M=$ $0.63, \mathrm{SD}=0.08$ ) group means were significantly larger than Democrat-controlled Senates $(M=0.48, \mathrm{SD}=0.11)$.

There was a significant difference in travel advisory policy group means for the Asian, Black, Latinx, and White ethnic groups. For the Asian ethnic group, the Tukey results showed that the case proportions in states with a travel advisory $(M=$ $0.05, \mathrm{SD}=0.01)$ group means were significantly larger than states without a travel advisory $(M=0.03, \mathrm{SD}=0.01)$. For the Black ethnic group, the Tukey results showed that the case proportions in states with no travel advisory $(M=0.08, \mathrm{SD}=$ 0.01 ) group means were significantly larger than states with a travel advisory $(M=0.06, \mathrm{SD}=0.01)$. For the Latinx ethnic group, the Tukey results showed that the case proportions in states with no travel advisory $(M=0.19, \mathrm{SD}=0.05)$ group mean were significantly larger than states with a travel advisory $(M=0.12, \mathrm{SD}=0.04)$. For the White ethnic group, the Tukey results showed that the case proportions in states with a travel advisory $(M=0.59, \mathrm{SD}=0.07)$ group mean were significantly larger than states with no travel advisory $(M=$ $0.51, \mathrm{SD}=0.07)$. Table 5 showed the beta analysis of variance results for the death proportions across ethnic groups.

There was a significant difference in the bar policy group means for the AIAN, Black, Latinx, and White ethnic groups. For the AIAN ethnic group, the Tukey results showed that death proportions for states with closed bars $(M=0.28$, SD $=0.05$ ) group mean were significantly larger than states with open bars $(M=0.21, \mathrm{SD}=0.05)$. For the Black ethnic group, the Tukey results showed that death proportions for states with open bars $(M=0.09, \mathrm{SD}=0.01)$ group mean were significantly larger than states with closed bars $(M=$ $0.04, \mathrm{SD}=0.01$ ). For the Latinx ethnic group, the Tukey results showed that death proportions for states with closed bars $(M=0.11, \mathrm{SD}=0.04)$ group mean were significantly larger than states with open bars $(M=0.07, \mathrm{SD}=0.02)$. For the White ethnic group, the Tukey results showed that death proportions for states with closed bars $(M=0.67$, SD $=0.08$ ) group mean were significantly larger than states with open bars $(M=0.59, \mathrm{SD}=0.08)$.

There was a significant difference in the curfew policy group means for the Blacks and NHPI ethnic groups. For the Black ethnic group, the Tukey results showed that death proportions for states with a curfew policy $(M=0.08, \mathrm{SD}$ $=0.01)$ group mean were significantly higher than states without a curfew policy $(M=0.05, \mathrm{SD}=0.01)$. For the NHPI ethnic group, the Tukey results showed that death proportions for states without a curfew policy group mean $(M=0.018$, $\mathrm{SD}=0.007)$ were significantly higher than states with a curfew policy $(M=0.013, \mathrm{SD}=0.006)$.

There was a significant difference in the governor's political affiliation group for the Asian and White ethnic groups. For the Asian ethnic group, the Tukey results showed that death proportions for states with a Democrat governor $(M=$ $0.04, \mathrm{SD}=0.01)$ group mean were significantly higher than states with a Republican governor $(M=0.03, \mathrm{SD}=0.009)$. For the White ethnic group, the Tukey results showed that death proportions for states with a Democrat governor $(M=$ $0.66, \mathrm{SD}=0.07)$ group mean were significantly higher than states with a Republican governor $(M=0.60, \mathrm{SD}=0.08)$.

There was a significant difference in the public school policy group means for the AIAN, Asian, and Black ethnic groups. For the AIAN group, the Tukey results showed that that death proportions for states that use a hybrid learning $(M=0.44, \mathrm{SD}=0.09)$ group mean were significantly larger than states that used virtual learning $(M=0.06, \mathrm{SD}=0.01)$. For the Asian ethnic group, the Tukey results showed that death proportions for states that use virtual learning $(M=$ $0.04, \mathrm{SD}=0.009$ ) group mean were significantly larger than states that used hybrid learning $(M=0.03, \mathrm{SD}=0.01)$. For the Black ethnic group, the Tukey results showed that that death proportions for states that use virtual learning $(M=$ $0.12, \mathrm{SD}=0.02)$ group mean were significantly larger than states that used hybrid learning $(M=0.01, \mathrm{SD}=0.02)$.

There was a significant difference in the restaurant policy group means for the Asian, Black, Latinx, and White ethnic groups. For the Asian ethnic group, the Tukey results showed that the death proportions for states with no policy $(M=0.02$, $\mathrm{SD}=0.009)$ group mean were significantly smaller than the closed restaurants $(M=0.04, \mathrm{SD}=0.01)$. For the Black ethnic group, the Tukey results showed that the death proportions for states that had no restaurant policies $(M=0.04$, $\mathrm{SD}=0.01)$ group mean were significantly smaller states with outdoor-only policies $(M=0.09, \mathrm{SD}=0.02)$, or the restaurants were closed $(M=0.07, \mathrm{SD}=0.02)$. For the Latinx ethnic group, the Tukey results showed that the death proportions for states that had outdoor-only restaurant policies 
Table 5 Death proportions beta analysis of variance results

\begin{tabular}{|c|c|c|c|c|c|c|c|c|c|}
\hline \multicolumn{4}{|l|}{ AIAN } & \multicolumn{3}{|c|}{ Asian } & \multicolumn{3}{|c|}{ Black } \\
\hline Source & $d f$ & $F$ Ratio & $p$-value & $\mathrm{df}$ & $F$ ratio & $p$-value & $d f$ & $F$ ratio & $p$-value \\
\hline Bars & 1 & 13.29 & $p<0.001$ & 1 & 9.38 & $p<0.01$ & 1 & 32.52 & $p<0.001$ \\
\hline Curfew & 1 & 2.92 & 0.088 & 1 & 3.18 & 0.074 & 1 & 28.79 & $p<0.001$ \\
\hline Governor & 1 & 1.64 & 0.200 & 1 & 6.95 & $p<0.01$ & 1 & 0.37 & 0.541 \\
\hline Mask mandate & 1 & 1.45 & 0.299 & 1 & 0.02 & 0.881 & 1 & 1.22 & 0.269 \\
\hline Public schools & 1 & 67.74 & $p<0.001$ & 1 & 1.08 & 0.300 & 1 & 96.49 & $p<0.001$ \\
\hline Restaurants & 2 & 2.18 & 0.113 & 2 & 4.26 & $p<0.05$ & 2 & 22.08 & $p<0.001$ \\
\hline State leg & 2 & 17.80 & $p<0.001$ & 2 & 3.47 & $p<0.05$ & 2 & 21.05 & $p<0.001$ \\
\hline State senate & 1 & 7.85 & $p<0.01$ & 1 & 0.80 & 0.370 & 1 & 7.87 & $p<0.01$ \\
\hline Travel advisory & 1 & 1.15 & 0.284 & 1 & 0.37 & 0.545 & 1 & 41.87 & $p<0.001$ \\
\hline \multicolumn{4}{|l|}{ Latinx } & \multicolumn{3}{|c|}{ NHPI } & \multicolumn{3}{|c|}{ White } \\
\hline Source & $d f$ & $F$ ratio & $p$-value & $d f$ & $F$ ratio & $p$-value & $d f$ & $F$ ratio & $p$-value \\
\hline Bars & 1 & 11.37 & $p<0.001$ & 1 & 1.60 & 0.206 & 1 & 10.16 & $p<0.01$ \\
\hline Curfew & 1 & 2.47 & 0.116 & 1 & 4.24 & $p<0.05$ & 1 & 3.77 & 0.052 \\
\hline Governor & 1 & 0.00 & 0.971 & 1 & 0.00 & 0.964 & 1 & 4.07 & $p<0.05$ \\
\hline Mask mandate & 1 & 0.14 & 0.706 & 1 & 1.93 & 0.164 & 1 & 0.65 & 0.419 \\
\hline Public schools & 1 & 0.06 & 0.800 & 1 & 0.00 & 0.979 & 1 & 2.32 & 0.128 \\
\hline Restaurants & 2 & 4.24 & $p<0.05$ & 2 & 0.90 & 0.409 & 2 & 15.62 & $p<0.001$ \\
\hline State leg & 2 & 3.67 & $p<0.05$ & 2 & 2.84 & 0.058 & 2 & 1.12 & 0.326 \\
\hline State senate & 1 & 3.70 & 0.054 & 1 & 0.23 & 0.634 & 1 & 8.36 & $p<0.01$ \\
\hline Travel advisory & 1 & 10.20 & $p<0.01$ & 1 & 0.28 & 0.594 & 1 & 21.80 & $p<0.001$ \\
\hline
\end{tabular}

There was a significant difference in state Senate legislature party majority group means for the AIAN, Black, and White ethnic groups. For the Asian ethnic group, the Tukey results showed that the death proportions in states with a Republican-controlled Senate $(M=0.04$, SD $=$ 0.01) group mean are significantly larger than Democratcontrolled Senates $(M=0.03, \mathrm{SD}=0.01)$. For the Black ethnic group, the Tukey results showed that the death proportions in states with a Democrat-controlled Senate $(M=$ $0.09, \mathrm{SD}=0.03)$ group mean are significantly larger than Republican-controlled Senates $(M=0.04, \mathrm{SD}=0.01)$. For the White ethnic group, the Tukey results showed that the death proportions in states with a Republican-controlled Senate $(M=0.73, \mathrm{SD}=0.07)$ group mean are significantly larger than Democrat-controlled Senates $(M=0.52, \mathrm{SD}=0.12)$.

There was a significant difference in travel advisory policy group means for the Black, Latinx, and White ethnic groups. For the Black ethnic group, the Tukey results showed that the death proportions in states with no travel advisory $(M=$ $0.09, \mathrm{SD}=0.01)$ group mean are significantly larger than states with a travel advisory $(M=0.04, \mathrm{SD}=0.01)$. For the Latinx ethnic group, the Tukey results showed that the death proportions in states with no travel advisory $(M=$ $0.11, \mathrm{SD}=0.03)$ group mean are significantly larger than states with a travel advisory $(M=0.07, \mathrm{SD}=0.02)$. For the White ethnic group, the Tukey results showed that the death 
proportions in states with a travel advisory $(M=0.69, \mathrm{SD}=$ $0.08)$ group mean are significantly larger than states with no travel advisory $(M=0.57, \mathrm{SD}=0.08)$.

\section{Discussion and conclusion}

The research effort aimed to investigate if the positive cases and death rates for POCs were higher than White ethnic groups. The research findings suggest that POC positive cases and death rates were higher in some states but not for the overall country. Whites consist of $76 \%$ of the total US and territories population based on the 2020 US Census data. It is expected that this ethnic group will have the highest percentage of total cases and deaths at the country population level. Regarding POCs at the country level, Latinxs have the highest rate of positive cases and Blacks have the highest rate of confirmed deaths. Blacks and Latinxs make up 35\% of the total positive cases and deaths among POCs in the US and territories.

Blacks are dying at a high rate in the southeastern states, District of Columbia, and Maryland. Specifically, in the District of Columbia, the death rate is five times higher than the White ethnic group. The states with both the highest proportion of positive cases and deaths for Blacks are the District of Columbia, Mississippi, and Georgia. For Latinx, the positive cases and death rates are mostly from western states, including Texas. Latinxs accounted for half the total deaths in Texas and California. Latinxs' death rate is higher than Whites in four states: Texas, California, New Mexico, and the District of Columbia. For AIAN, Alaska, New Mexico, Montana, and South Dakota have the highest rates of cases and deaths. Their death rate, when compared to Whites, is the highest in New Mexico and Alaska. For both Asian and NHPI ethnics groups, positive cases and deaths are the highest in Hawaii and Guam. These results are because they make up the dominant nationality in that state and territory. Compared to Whites, the death rate is 30 to 1 , but it has been concluded that the impact is practically significant due to the overall low probability in cases and deaths countrywide. Our research findings are consistent with studies done by [16] and [21] which suggest that POC positive cases and death rates were higher in some states but not for the overall country. Specifically, in the District of Columbia, the death rate of Blacks was five times higher than the White ethnic group, while in Michigan, their death rate was three times higher than the White ethnic group [21].

The second aim of the research effort was to investigate a significant difference in confirmed positive cases and death rates between ethnic groups across the US and territories. Our findings suggest a significant difference in positive cases and death rates across the US and territories. The most concerning results are the states clustered both in the highest rate of cases and deaths for a particular ethnic group, specifically Blacks and Latinxs. For Blacks, these states are Alabama, District of Columbia, Delaware, Georgia, Louisiana, Maryland, Mississippi, South Carolina, and Virginia. For Latinxs, these states are Arizona, California, Colorado, Florida, New Jersey, New Mexico, and Texas. The other POCs' rates are not critical based on the data used in this research effort. These results are consistent with the studies done by [6, 14, 28].

The third aim was to investigate if political party control and governmental policies affected case and death proportion rates across ethnic groups. There were significant findings found in the rate of deaths and cases per ethnic group for policies and political factors except for the mask mandate policy. The research suggests that mask mandates were not a factor in the cases or death rates of any ethnic group based on the data analyzed. However, the research by [4, 31] suggested that mask mandates reduced infection rate and the spread of COVID-19. The policies and political factors that had the most influences across ethnic groups were the bar policy, curfew policy, public school policy, travel advisory policy, and political party control of each state congress. The results for the death and case proportions due to implemented policy varied between ethnic groups. However, the research suggested that policies are an important factor concerning the rate of cases and deaths. These results are similar to the other research efforts indicating that stay-at-home orders (curfews) were important in reducing the COVID-19 infection rate in the USA. Elena et.al. found that school closure had a significant impact on infection rate and strict travel bans in China proved to be an important policy that reduced the spread of COVID-19 [22].

A limitation of the current research is that the data for unknown ethnicity of cases and deaths were not used. The exclusion of unknown nationalities could affect the overall results due to POC low sample sizes. This limitation is especially true for the small number of datasets for AIAN, Asian, and NHPI outside Hawaii and Guam. Also, the small factor group sizes only having one state with the split controlled state legislature and two states with hybrid public schools' policies from during the data collection period.

Another limitation of this research effort is that key factors such age profiles, socioeconomic factors, and preexisting medical conditions were not provided. These key factors would provide more insight on the ethnic disparities in cases and deaths. Adams and her colleagues stated that lack of insurance is a major issue among POC communities [1]. Individuals who struggle with income stability are less likely to have health insurance. Inadequate or nonexistent insurance will lead to a decreased likelihood of seeking healthcare due to concerns of medical cost. Specifically for African Americans, diabetes, hypertension, and heart disease are prevalent throughout the community. Due to the higher risk of death 
from COVID-19, efforts to investigate the ethnic disparities due to preexisting condition and age are warranted.

Based on the research findings, it is necessary to develop a more holistic business-education system (system-of-system) that can provide communities with appropriate public health, identify future interventions, and offer a deeper understanding of the factors that influence the spread of diseases.

In effect, the challenges of COVID-19 and other potential similar diseases are likely to escalate in the future as we grapple with the interdisciplinary system problems of the twenty-first century. Clearly, there is an immediate need to develop more systematic business models that integrate the technical, social, organizational, managerial, policy, and political factors in the US healthcare system.

Further research should be conducted at the state level for the states listed above for Blacks and Latinxs as well as an investigation into the county-level relationships. Once the results are acquired, the social-cultural analysis should be conducted at the state and county levels. This should include investigating whether the rates of cases and deaths are due to social variables such as mask mandates, state lockdowns, business closure periods, and other social variables. Models should be developed to investigate the patterns of social and policy behaviors on rates of deaths across POCs.

\section{References}

1. Adams, M.L., Katz, D.L., Grandpre, J.: Population-based estimates of chronic conditions affecting risk for complications from coronavirus disease, United States. Emerg. Infect. Dis. 26(8), 1831-1833 (2020). https://doi.org/10.3201/eid2608.200679

2. Balmford, B., Annan, J.D., Hargreaves, J.C., et al.: Cross-country comparisons of Covid-19: policy, politics, and the price of life. Environ. Resour. Econ. 76, 525-551 (2020). https://doi.org/10.10 07/s10640-020-00466-5

3. Ballotpedia-Composition of Governors and state legislators. https://ballotpedia.org/Partisan_composition_of_state_ legislatures

4. Brian, N., et al.: Associations between Governor Political Affiliation and COVID-19 cases, deaths, and testing in the US. Am. J. Prev. Med. (2021). https://doi.org/10.1016/j.amepre.2021.01.034

5. Bring your data to life with Microsoft Power BI". Microsoft.com. Microsoft. Retrieved May 4, 2016. https://powerbi.microsoft.com/ en-us/

6. Brown, K.M., et al.: An ecological study of the association between neighborhood racial and economic residential segregation with COVID-19 vulnerability in the United States capital city. Ann. Epidemiol. 59, 33-36 (2021)

7. Castex, G., Dechter, E., Lorca, M.: COVID-19. The impact of social distancing policies, cross-country analysis. EconDisCliCha (2020). https://doi.org/10.1007/s41885-020-00076-x

8. Chen, N., Zhou, M., Dong, X., Qu, J., Gong, F., Han, Y., et al.: Epidemiological and clinical characteristics of 99 cases of 2019 novel coronavirus pneumonia in Wuhan, China: a descriptive study. Lancet 395(10223), 507-513 (2020). https://doi.org/10.1016/S01 40-6736(20)30211-7

9. Cribari-Neto, F., Zeileis, A.: Beta Regression in R. J. Stat. Softw. 34(2). www.jstatsoft.org/article/view/v034i02/v34i02.pdf (2010)
10. Curfew mandate-https://www.finra.org/rules-guidance/key-topics/ covid-19/shelter-in-place

11. Dong, E., Du, H., Gardner, L.: An interactive web-based dashboard to track COVID-19 in real-time. Lancet Inf Dis. 20(5), 533-534 (2020). https://doi.org/10.1016/S1473-3099(20)30120-1

12. Dorn, A.V., Cooney, R.E., Sabin, M.L.: COVID-19 exacerbating inequalities in the US. Lancet 395(10232), 1243-1244 (2020). https://doi.org/10.1016/S0140-6736(20)30893-X

13. Hsiang, S., Allen, D., Annanan, S., et al.: The effect of large-scale anti-contagion policies on the COVID-19 pandemic. Nature 584, 262-267 (2020)

14. Hawkins, R.B., et al.: Socio-economic status and COVID-19 related cases and fatalities. J. Pub. Health 189, 129-134 (2020)

15. Kassambara, A., Mundt, F.: Extract and Visualize the Results of Multivariate Data Analyses", R package version 1.0.7. https:// CRAN.R-project.org/package $=$ factoextra

16. Lee, W., et al.: Urban Environments and COVID-19 in three Eastern states in the United States. Sci. Total Environ. 779, 146334 (2021)

17. Liang, L.L., Tseng, C.H., Ho, H.J., et al.: Covid-19 mortality is negatively associated with test number and government effectiveness. Sci. Rep. 10, 12567 (2020). https://doi.org/10.1038/s41598020-68862-x

18. MacQueen, J: Some methods for classification and analysis of multivariate observations. In: Proceedings of the Fifth Berkeley Symposium on Mathematical Statistics and Probability, Volume 1: Statistics, Berkeley, Calif.: University of California Press. pp. 281-97 (1967). http://projecteuclid.org:443/euclid.bsmsp/120 0512992

19. NewYorkTimespoliciesacrossstates.https://www.nytimes.com/ interactive/2020/us/states-reopen-map-coronavirus.html

20. Parodi, S.M., Liu, V.X.: From containment to mitigation of COVID-19 in the US. JAMA 323(15), 1441-1442 (2020). https:// doi.org/10.1001/jama.2020.3882

21. Parpia, A.S., et al.: Racial disparities among COVID-19 mortality in Michigan, United States. EClinnical Med. 33, 100761 (2021)

22. Renan, C.C., et al.: The effect of state-level stay-at-home orders on COVID-19 infection rates. Am. J. Infect. Control 48(8), 958-960 (2020). https://doi.org/10.1016/j.ajic.2020.05.017

23. R Core Team. R: A language and environment for statistical computing. R Foundation for Statistical Computing, Vienna, Austria (2020). https://www.R-project.org/

24. School mandate-https://www.edweek.org/leadership/mapcoronavirus-and-school-closures-in-2019-2020/2020/03

25. Staguhn, D.E. et al.: The impact of statewide school closures on COVID-19 infection rates. Am. J. Infect. Control

26. Travelrestrictions-https://www.nashp.org/governors-prioritizehealth-for-all/

27. Tibshirani, R., Walther, G., Hastle, T.: Estimating the number of clusters in a data set via the gap statistic. J. R. Stat. Soc. B 63(Part 2), 411-423 (2001)

28. Tirupathi, R., et al.: COVID-19 disparity among racial and ethnic minorities in the US: a cross-sectional analysis. Travel Med. Infect. Dis. 38, 101904 (2020)

29. U.S. Census Bureau: United States QuickFacts for Race and Hispanic Orgin (2020). https://www.census.gov/quickfacts

30. Van Nguyen, Q., et al.: Spread of COVID-19 and policy responses in Vietnam: an overview. Int. J. Infect. Dis. 103, 157-161 (2021)

31. Xue, L., et al.: Evaluating the impact of the travel ban within mainland China on the epidemic of the COVID-19. Int. J. Infect. Dis. (2021). https://doi.org/10.1016/j.ijid.2021.03.088

Publisher's Note Springer Nature remains neutral with regard to jurisdictional claims in published maps and institutional affiliations. 